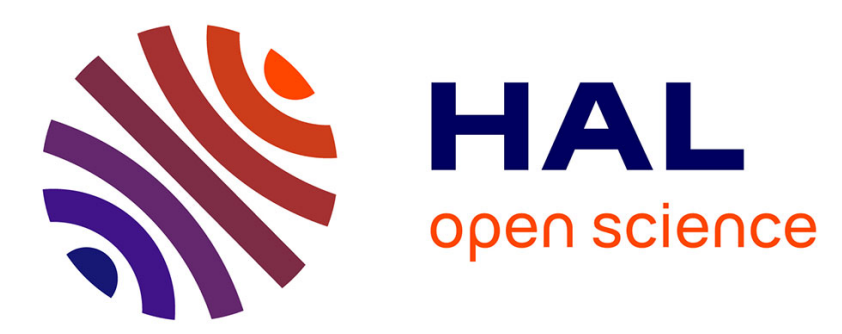

\title{
Graph Modelling of Musical Wind Instruments: A Method for Natural Frequencies Computation G Le Vey
}

\section{To cite this version:}

G Le Vey. Graph Modelling of Musical Wind Instruments: A Method for Natural Frequencies Computation. Acta Acustica united with Acustica, 2015, 101 (6), pp.1222-1233. 10.3813/AAA.918915 . hal-01312797

\section{HAL Id: hal-01312797 \\ https://hal.science/hal-01312797}

Submitted on 9 May 2016

HAL is a multi-disciplinary open access archive for the deposit and dissemination of scientific research documents, whether they are published or not. The documents may come from teaching and research institutions in France or abroad, or from public or private research centers.
L'archive ouverte pluridisciplinaire HAL, est destinée au dépôt et à la diffusion de documents scientifiques de niveau recherche, publiés ou non, émanant des établissements d'enseignement et de recherche français ou étrangers, des laboratoires publics ou privés. 


\title{
Graph modelling of musical wind instruments : a method for natural frequencies computation
}

\author{
G. Le Vey \\ Ecole des Mines de Nantes, IRCCyN UMR-CNRS 6597 \\ 4 , rue A. Kastler, 44300 NANTES, FRANCE \\ levey@emn.fr
}

\begin{abstract}
Many aspects of the musical wind instruments behavior can be studied within the framework of 1D acoustic propagation. A common method is based on an analogy with electric transmission lines, in which the impedance is a central concept. The present work proposes a different approach, based on results from mathematical modelling and analysis of repetitive structures such as networks of strings or beams. The method, that keeps at the one dimensional level, uses concepts and methods from graph theory for modelling the duct of wind instruments. A key point is a special matrix reformulation of the original wave equation on a graph. The focus is on natural frequencies computations for complex, piecewise cylindrical ducts. Examples with or without toneholes illustrate the method and its potential through symbolic computations. In the case of closed-open piecewise cylindrical resonators, the approach answers in a practical way a conjecture formulated twenty years ago by Dalmont and Kergomard, which is then replaced in the general framework of inverse spectral problems. An assertion of Benade is checked on one woodwind example.
\end{abstract}

\section{Introduction}

Wind instruments have been the subject of numerous studies for a long time and are now rather well understood $[1,2,3,4]$. A wind instrument can be considered in the first approximation as a duct, the length of which can be made variable by using some device : either by opening or closing one or several toneholes for woodwinds ; or adjusting by other means the overall length of the duct for brasses, thereby adjusting the pitch of the instrument. Apart from the more complex finite elements computations, the transmission lines formalism is of common use $[2,4,5,6,7]$ : it has low complexity, using only simple matrix calculus, and has shown its efficiency for numerous questions in acoustical 1D propagation, either musical or not, mainly through input impedance calculations.

In the present work, a modelling approach to musical wind instruments is proposed, not using the transmission lines analogy. Its basic element is also the 1D wave equation, but written on a graph that describes the topology of a wind instrument. For this reason, similarities can surely be found with the transmission lines-based approach but this point is not touched upon here as it needs more investigation. Notice also that because it remains at $1 \mathrm{D}$ level, complex phenomena at junctions cannot be accounted for at this 
stage. The method allows to model in a flexible way resonators with variable crosssection and with or without toneholes. But its algorithmic complexity remains low, at the price of using a mathematical formulation that looks unusual in the acoustics community. Designing ducts that have harmonically related frequencies is a key point for musical wind instruments thus the focus in this work is on natural frequencies computation under the linear acoustics assumption (a first theoretical presentation is in [8]). In some situations, eigenmodes can be computed at once with the eigenvalues. For simplicity, the exposition restricts to piecewise cylindrical resonators. Neither losses nor radiation impedance are included at this stage, in spite of their importance for natural frequencies computation : only ideal boundary conditions are considered.

A first issue in the study of wind instruments concerns the duct cross-section. It is known that the duct must have harmonically related natural frequencies for intonation and stability reasons [2]. It is also generally taken for granted that the musically useful continuous ducts are approximately members of the Bessel horn family, including cones and cylinders [2,9]. Practical instruments show that the duct is not precisely cylindrical nor conical and that small variations from these idealized shapes arise, e.g. from deliberate alterations brought by the instrument maker in order to improve the tone or the tuning of an instrument [7, 10, 11]. For ducts with discontinuities, a family of piecewise cylindrical resonators, called stepped cones, closed at one end and open at the other one (models of reed instruments), has been shown in [12] to have harmonically related natural frequencies, provided their geometry satisfies some conditions. It was conjectured [12] that no other resonators share this property.

A second issue is that the acoustical behavior of woodwinds is known to be strongly influenced by the design of its system of toneholes : their sizes and spacings cannot be designed independently for a tube of a given thickness [10]. In particular, the presence of toneholes affects the natural frequencies of the whole instrument $[13,14]$. Therefore, A.H. Benade proposed [2] to look at a woodwind as a sequence of 'Tshaped sections', each consisting of a piece of the main duct and one tonehole, with given radii and lengths. In order to obtain qualitative results, a simplified version concatenates identical such T-shaped sections, as a kind of periodic medium. This results in approximate formulae that can be used for design [2, 15]. Several effects were studied such as those due to the closed-holes or open-holes length corrections, to fork fingerings, or to the function of register hole for higher register functioning ([2] and [4], chap. 7 for a survey). The main objectives are to obtain information about the playing frequencies and how they are influenced by the toneholes geometry. This approach has been the main basis of most works since (see [13, 14, 16] e.g.).

Both above keypoints are studied within the proposed approach, through two applications in section 6 : piecewise cylindrical resonators and wind instruments with one, two and three toneholes, without regularity assumptions on the geometry. The main ingredient comes from mathematical studies of equations on networks [17, 18, 19] and more recently on assemblages of several similar components such as strings, beams, plates (see [20] and references therein). The basic steps are : 1) describe the graph of this network connecting together the elementary components ; 2) account for conservation laws at the junctions ; 3) study the spectral characteristics of this set through properties of the graph itself and of the components, thanks to a convenient reformulation. This way, the one dimensional setting, useful for numerous questions related to musical wind instruments, is kept although complex geometries are in order, without going through full 3D computations. As a consequence the method does not describe complex phenomena at the junctions between several ducts.

First numerical results using this approach have been presented in [21]. The method 
has shown the interest of having at hand closed-form expressions for the design of piecewise cylindrical resonators [22] and to help when using optimization procedures for design [23]. Thus, in the present work, the accent is put on what makes a difference with the classical approach : closed-form expressions and symbolic computations. A byproduct of the approach is a practical answer given to the conjecture of [12] we mentionned above, which is also briefly replaced in the more general mathematical setting of inverse spectral problems [24]. An assertion of A.H. Benade [2] about the influence of open toneholes beyond the second or third ones is checked on an example.

The presentation is organized as follows : section 2 presents the geometric description of a woodwind as a network of elementary components and section 3 completes it to the full model with its physical properties. Section 4 is dedicated to the theoretical part of the proposed method for computing the natural frequencies, with the main result given in section 4.3, along the lines of [19]. In sections 5 and 6 two applications with progressive complexity : piecewise cylindrical resonators and very simple woodwinds with one, two and three toneholes illustrate the potential of the method.

\section{Geometry of wind instruments}

For modelling wind instruments with graph theory, the general idea is the following : consider a simplistic woodwind as in figure 1. The case of resonators without toneholes is even simpler. The main duct and its toneholes are modelled by edges of a graph (see figure 2). They meet at vertices that model their junction. As precised at the end of this section, the edges are oriented to account for the chosen velocity convention. Consider

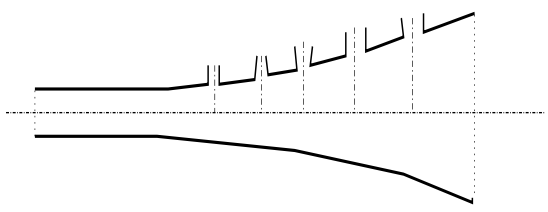

Figure 1: scheme of a woodwind

also that the main duct is not made of one piece but of several ones connected in a serial fashion. Their interconnection is done where the edge modelling one tonehole connects too or where a change in cross-section occurs. One cylindrical portion can be divided into smaller pieces, e.g. in a process of discretization. This gives some flexibility when considering varying cross section instruments, even when only cylinders are considered as basic components of the graph as here. Then the union of all these edges and vertices constitutes the underlying graph $\mathbf{G}$ of the instrument [8]. Such a graph is of a very special type : it is a tree as it is connected and contains no cycle [25]. The resulting tree of a general woodwind is illustrated in figure $2:$ for a wind instrument with $h$ holes, the associated tree has $N=2 h+1$ edges, denoted $E_{i}$ and $N+1=2 h+2$ vertices, denoted $V_{i}$. Such a labeling is obviously arbitrary and any other suits. But it was chosen for including boundary conditions more easily (see section 4.4). Notice that for a piecewise continuous resonator without toneholes, edges $E_{h+2}, E_{h+3}, \ldots, E_{2 h+1}$ are merely suppressed (see figure 2). Even if the duct is continuous, a sufficiently fine discretization in cylinders still makes the approach useful for non uniform ducts. Each edge and its associated quantities are indexed by an integer in the set : $\mathcal{T}=\{1, \ldots, N\}$. Therefore define for each edge labelled $j \in \mathcal{J}$, the length $L_{j}$, the running variable 


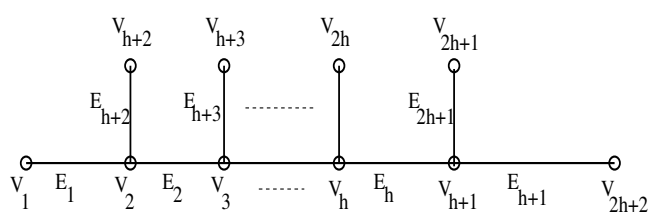

Figure 2: Graph of a woodwind with toneholes

$x_{j} \in\left[0, L_{j}\right]$, the cross-section area $a_{j}$, the pressure $p_{j}$, the particle velocity $v_{j}$ and the velocity potential $\varphi_{j}\left(v_{j}=\partial_{x_{j}} \varphi_{j}\right)$. In the most general case, the sound velocity, $c_{j}$, is different in each tube, although this is likely not to be the usual case. Notice however that for mouth-blown instruments, a gradient of temperature and hygrometry may exist between both ends, that could imply a non constant sound velocity along the duct. The locations of end points of each tube, i.e. the vertices of the tree, are labelled by an integer in the set : $\mathcal{I}=\{1, \ldots N+1\}$. Figure 2 shows that simple vertices belong to the boundary of the graph $\mathbf{G}$ and that multiple vertices belong to its interior. For $i \in \mathcal{I}$, define : $\mathcal{I}_{i}=\{j \in \mathcal{J}$ : the $j$ th tube meets the $i$ th vertex $\}$. For $j \in \mathcal{I}_{i}$, define the binary variable $x_{i j}$ such that $x_{i j}=0$ or $L_{j}$ to identify which end meets the other tubes at the $i$ th vertex. Set also $d_{i j}=1$ if $x_{i j}=L_{j}$ and $d_{i j}=-1$ if $x_{i j}=0$, to account for orientation.

\section{Linear physical model of a wind instrument}

The above geometrical description of a wind instrument has to be completed by physical properties, assumed to conform to the plane wave lossless linear approximation. The usual assumptions for propagation in ducts without sources are in order in the sequel : the fluid is barotropic i.e. the pressure is a function of the density $\rho$ only : $p=p(\rho)$ and the quantities of interest are the small perturbations of variables about their mean values. In the sequel, $c$ is the velocity of sound in free space, $p(x, t)$ the pressure, $v(x, t)$ the volume velocity, $a(x)$ the cross-section area of a tube at abcissa $x$, all indexed by $j \in \mathcal{J}$. The fluid is assumed irrotational, i.e. there exists a velocity potential $\varphi: v=\partial_{x} \varphi$. Under these assumptions, the horn wave equation models the propagation in each tube :

$$
\frac{1}{c_{j}^{2}} a_{j} \partial_{t t} \varphi_{j}-\partial_{x_{j}}\left(a_{j} \partial_{x_{j}} \varphi_{j}\right)=0, j \in \mathcal{J}
$$

The interconnection is modelled by a conservation law at each node. Although other laws could be considered, Kirchoff-type flow conservation laws at each multiple node are customary and are in order :

$$
\sum_{j \in \mathcal{I}_{i}} d_{i j} a_{j} \partial_{x_{j}} \varphi_{j}\left(x_{i j}, t\right)=0, \forall i \in \mathcal{I}
$$

This reduces to a Neumann condition at a simple node $i$ connected to the sole edge $j: \partial_{x_{j}} \varphi_{j}\left(x_{i j}, t\right)=0$ corresponding to an ideally closed hole. An ideally open hole imposes the Dirichlet condition : $\varphi_{j}\left(x_{i j}, t\right)=0$. All the above represents a lossless wind instrument without active components.

Remark Within the present approach and still in the linear acoustics framework, some dissipation phenomena can be taken into account, at least formally, through an 
additional term proportional to a first order time derivative operating on the laplacian, leading to a complex generalized sound velocity. Its real part represents the true sound velocity and the imaginary part the damping. The resulting equation can then be inserted into the same graph theory framework but the consequences on the solution method hereafter has not yet been studied. Another possible way is to consider 1D model for elementary ducts such as those developed in [26] that can include viscothermal losses. Radiation impedance neither is included : as it is frequency-dependent, it is usually formulated in the frequency domain. Nevertheless, including a length correction to the last duct is a simple way to take it into account approximately [4] within the proposed approach. In this work, boundary conditions at the end of the ducts have been taken ideal : either the pressure or the volume velocity vanish at the ends of the instrument.

\section{Natural frequencies of a wind instrument : theory}

Determining the natural frequencies of a wind instrument -i.e., in mathematical terms, the spectrum of the differential operator defined in (1), (2) with boundary conditionsis central from the viewpoints of physics, of instrument making and is important for musical practice, as it was recalled in the introduction. In that context, the graph modelling approach presented above can be an alternative method to the transmission lines approach for computing the natural frequencies, as it consists in one dimensional equations, far less complex than full 3D models. However, being one dimensional, the method is intrinsically an approximate one and at this stage complex phenomena at junctions are not described. The main point to remember is that the geometrical structure of an instrument is evidenced through matrices attached to the underlying graph. One important aspect of the method is to rescale the spatial variables associated with each edge of the graph to the unit interval $[0,1]$, while choosing an orientation on each edge. Then an elementwise matrix calculus, attributed to J. Hadamard, allows to pose and solve the corresponding eigenvalue problem. In summary, the method proceeds along the following steps : first build a full characteristic matrix by considering the geometry of the underlying graph. Then introduce boundary conditions in order to possibly reduce this characteristic matrix. Eventually, compute the natural frequencies from the resulting eigenvalue problem. These necessary steps are given below with the main result in section 4.3. Not only the natural frequencies but also, in some instances, an approximation of the eigenmodes at the vertices of the graph can be computed.

\subsection{The eigenvalue problem on the graph}

Firstly, using equations (1), (2) in section 3, the natural frequencies $\omega=2 \pi f$ of a woodwind model are the nontrivial solutions of the following eigenvalue problem :

$$
\left\{\begin{array}{l}
\varphi_{j} \in C^{2}\left(\left[0, L_{j}\right]\right), \forall j \in \mathcal{J}, \varphi=\left(\varphi_{j}\right) \\
\varphi \text { is continuous on } \mathbf{G} \\
\partial_{x_{j} x_{j}} \varphi_{j}+\frac{\partial_{x_{j}} a_{j}}{a_{j}} \partial_{x_{j}} \varphi_{j}=-\frac{\omega^{2}}{c_{j}^{2}} \varphi_{j}, \forall j \in \mathcal{J} \\
\sum_{j \in \mathcal{I}_{i}} d_{i j} a\left(x_{i j}\right) \partial_{x_{j}} \varphi_{j}\left(x_{i j}, t\right)=0, \forall i \in \mathcal{I}
\end{array}\right.
$$

This system is nothing else than gathering wave horn equations in all the elementary parts together with the conservation laws at the junctions. In this form however, this boundary value problem, although linear, is too complex to solve : the differential 
equations are defined on different intervals and the boundary conditions given by the conservation laws are not easy to deal with. It is best to reformulate it with a view to another resolution method.

\subsection{A matricial reformulation}

The purpose of this section, following [19] that treated a parabolic problem, is to transform the nonstandard boundary-value, vector eigenvalue problem (3) into a standard initial-value, matrix one that gives directly the searched after solution, which would be very difficult by other means. Two tools are necessary to this end. The first one is a mere rescaling of all variables onto the unit interval $[0,1]$, by setting $L_{j} \xi=x_{j}$. In that case, $x_{j} \in\left[0, L_{j}\right], \forall j \in \mathcal{J} \Rightarrow \xi \in[0,1]$. The second one is a matrix reformulation of problem (3), for which the following notations and definitions are needed. Consider the tree (see figure 2), $\mathbf{G} \subset \mathbb{R}^{2}$, of a woodwind having $h$ toneholes, with its set of $N=2 h+1$ edges, $E(\mathbf{G}):=\left\{E_{j}, j \in \mathcal{J}\right\}$ and its set of $N+1=2 h+2$ vertices, $V(\mathbf{G}):=\left\{V_{i}, i \in \mathcal{I}\right\}$. The edges are parameterized by $\pi_{j}:\left[0, L_{j}\right] \rightarrow \mathbb{R}^{2}$, where the running variable $x_{j} \in\left[0, L_{j}\right]$ represents the arc length. The maps $\pi_{j}$ are assumed to be $C^{2}$-smooth. Introduce the $(N+1) \times N$ incidence matrix $\mathcal{D}=\left(d_{i j}\right), i \in \mathcal{I}, j \in \mathcal{J}$ :

$$
d_{i j}=\left\{\begin{aligned}
1 & \text { if } V_{i} \text { is the end of edge } j \\
-1 & \text { if } V_{i} \text { is the origin of edge } j \\
0 & \text { otherwise }
\end{aligned}\right.
$$

which puts in matrix form the $d_{i j}$ 's introduced in section 2 : it describes the orientation chosen on the edges of the graph. The $(N+1) \times(N+1)$ adjacency matrix $\mathcal{E}=$ $\left(e_{i_{1} i_{2}}\right), i_{1}, i_{2} \in \mathcal{I}$ :

$$
e_{i_{1} i_{2}}= \begin{cases}1 & \text { if } V_{i_{1}} V_{i_{2}} \text { is an edge } \\ 0 & \text { otherwise }\end{cases}
$$

describes how edges connect vertices. Let $s\left(i_{1}, i_{2}\right)$ be the integer labeling the edge connecting vertices $V_{i_{1}}$ and $V_{i_{2}},\left(i_{1}, i_{2} \in \mathcal{I}\right)$. As mentionned above, this labeling is up to a permutation of the set $\mathcal{I}$ an it is obviously symmetric : $s\left(i_{1}, i_{2}\right)=s\left(i_{2}, i_{1}\right)$. For mathematical completeness, whenever $e_{i_{1} i_{2}}=0$ (i.e. no edge connects vertices $i_{1}$ and $\left.i_{2}\right), s\left(i_{1}, i_{2}\right)$ can be set to any value, say $s\left(i_{1}, i_{2}\right)=1$, as it will not come into play in the equations. The ordinary product of matrices $N^{1}, N^{2}$, having compatible dimensions, is denoted as usual by a simple concatenation $N^{1} N^{2}$. Let now $M^{1}, M^{2}$ be matrices with identical dimensions. Their Hadamard product, denoted $M^{1} \star M^{2}$, is defined elementwise by : $\left(M^{1} \star M^{2}\right)_{i_{1} i_{2}}=m_{i_{1} i_{2}}^{1} m_{i_{1} i_{2}}^{2}$ and is obviously commutative. For any function $f: \mathbb{R} \rightarrow \mathbb{R}$, and a matrix $M$ defined on the graph, the matrix $f(M)$ is also defined elementwise by $f(M)=\left(f_{i_{1} i_{2}}\right)$ with :

$$
f_{i_{1} i_{2}}=\left\{\begin{aligned}
f\left(m_{i_{1} i_{2}}\right) & \text { if } e_{i_{1} i_{2}}=1 \\
0 & \text { if } e_{i_{1} i_{2}}=0
\end{aligned}\right.
$$

and especially when $f(x)=x^{r}, r \in \mathbb{R}$, the matrix powers in the Hadamard sense are denoted $M^{(r)}$. Define the $N+1$-dimensional vector $z=(1, \ldots, 1)^{T}$ and, for any vector $y$, the diagonal matrix $\operatorname{diag}(y)$ with main diagonal made of the elements of $y$. Remembering the notations of section 3, define the matrices :

$$
\mathcal{A}=\left(a_{s\left(i_{1}, i_{2}\right)} e_{i_{1} i_{2}}\right), C=\left(c_{s\left(i_{1}, i_{2}\right)} e_{i_{1} i_{2}}\right), \mathcal{L}=\left(L_{s\left(i_{1}, i_{2}\right)} e_{i_{1} i_{2}}\right)
$$


that have the same pattern as $\mathcal{E}$ but carry the information about cross-sections, sound velocities and lengths respectively for each edge. Also, for $\varphi: \mathbf{G} \rightarrow \mathbb{R}$ and $\xi \in[0,1]$, define the matrix $\Phi(\xi)=\left(\phi_{i_{1} i_{2}}(\xi)\right)$ with :

$$
\phi_{i_{1} i_{2}}(\xi)= \begin{cases}\varphi_{s\left(i_{1}, i_{2}\right)}\left(L_{s\left(i_{1}, i_{2}\right)}(1-\xi)\right) & \text { if } V_{i_{1}} \text { is the end of edge } s\left(i_{1}, i_{2}\right) \\ \varphi_{s\left(i_{1}, i_{2}\right)}\left(L_{s\left(i_{1}, i_{2}\right)}\right) & \text { if } V_{i_{1}} \text { is the origin of edge } s\left(i_{1}, i_{2}\right) \\ 0 & \text { otherwise }\end{cases}
$$

It is a matrix version of the dependent variable, the potential $\varphi$. One has in particular : $\Phi(0)=\left(\psi z^{T}\right) \star \mathcal{E}, \psi$ denoting the vector of values of $\varphi$ at the vertices. Notice the symmetry $\phi_{i_{2} i_{1}}(\xi)=\phi_{i_{1} i_{2}}(1-\xi), \xi \in[0,1]$. By construction, $\Phi$ contains in a redundant form the information about the original potential $\varphi$. It is this redundancy that allows to transform the original boundary value problem (3) into an initial value one (12) below, leading to a closed-form solution (14). Because these notations are not easy to grasp at first reading, let us give an example. For a duct with three serially connected cylinders, without tonehole (see figure 2), there are $N=3$ edges labelled 1 to 3 and oriented from left to right, and 4 vertices labelled 1 to 4 from left to right. Thus : $s(1,2)=1, s(2,3)=2, s(3,4)=3$ and $s\left(i_{1}, i_{2}\right)=0$ otherwise. Matrices $\mathcal{D}, \mathcal{E}, \mathcal{A}, \mathcal{L}$, for this labeling, are given below while the non vanishing generic elements of matrix $\Phi$ followed by their value at the vertices are detailed. Notice from the latter how the original boundary value problem is transformed into an initial value one.

$$
\begin{aligned}
& \mathcal{D}=\left(\begin{array}{ccc}
-1 & 0 & 0 \\
1 & -1 & 0 \\
0 & 1 & -1 \\
0 & 0 & 1
\end{array}\right) \quad \mathcal{E}=\left(\begin{array}{llll}
0 & 1 & 0 & 0 \\
1 & 0 & 1 & 0 \\
0 & 1 & 0 & 1 \\
0 & 0 & 1 & 0
\end{array}\right) \\
& \mathcal{A}=\left(\begin{array}{cccc}
0 & a_{1} & 0 & 0 \\
a_{1} & 0 & a_{2} & 0 \\
0 & a_{2} & 0 & a_{3} \\
0 & 0 & a_{3} & 0
\end{array}\right) \mathcal{L}=\left(\begin{array}{cccc}
0 & L_{1} & 0 & 0 \\
L_{1} & 0 & L_{2} & 0 \\
0 & L_{2} & 0 & L_{3} \\
0 & 0 & L_{3} & 0
\end{array}\right) \\
& \phi_{12}(\xi)=\varphi_{1}\left(L_{1} \xi\right), \phi_{21}(\xi)=\varphi_{1}\left(L_{1}(1-\xi)\right), \quad \phi_{12}(0)=\varphi_{1}(0), \phi_{21}(0)=\varphi_{1}\left(L_{1}\right), \\
& \phi_{23}(\xi)=\varphi_{2}\left(L_{2} \xi\right), \phi_{32}(\xi)=\varphi_{2}\left(L_{2}(1-\xi)\right), \quad \phi_{23}(0)=\varphi_{2}(0), \phi_{32}(0)=\varphi_{2}\left(L_{2}\right), \\
& \phi_{34}(\xi)=\varphi_{3}\left(L_{2} \xi\right), \phi_{43}(\xi)=\varphi_{3}\left(L_{3}(1-\xi)\right) \quad \phi_{34}(0)=\varphi_{3}(0), \phi_{43}(0)=\varphi_{3}\left(L_{3}\right)
\end{aligned}
$$

Last, due to the normalization on the unit interval, the spatial derivatives can be denoted with primes $\left(u^{\prime}=\partial_{\xi} u\right)$ hereafter. With the above notations, the vector eigenvalue problem (3) can be shown, after some computations, to be equivalent to the following matrix one (taking care that $\Phi$ is a matrix, not a vector) :

$$
\left\{\begin{array}{l}
\phi_{i_{1} i_{2}} \in C^{2}([0,1]) \forall i_{1}, i_{2} \in \mathcal{I} \\
\left(e_{i_{1} i_{2}}=0 \Rightarrow \phi_{i_{1} i_{2}}=0\right) \\
\mathcal{L}^{(-2)} \star C^{(2)} \star \Phi^{\prime \prime}(x)+\mathcal{L}^{(-1)} \star C^{(2)} \star \mathcal{A}^{(-1)} \star \mathcal{A}^{\prime} \star \Phi^{\prime}(x)=-\omega^{2} \Phi(x), \forall x \in[0,1] \\
\exists \psi \in \mathbb{R}^{N+1}: \Phi(0)=\left(\psi z^{T}\right) \star \mathcal{E} \\
{\left[\mathcal{L}^{(-1)} \star \mathcal{A} \star \Phi^{\prime}(0)\right] z=0} \\
\Phi^{T}(\xi)=\Phi(1-\xi), \forall \xi \in[0,1]
\end{array}\right.
$$

The nontrivial solutions $\omega$ of this matrix eigenvalue problem equivalently furnish the natural frequencies of the modelled wind instrument. A detailed analysis within this generality is deferred to future work. Instead, an important particular case will illustrate the method while giving new results. It is firstly reasonable to assume that all the $c_{i}$ 's 
are equal to the same constant $c$ so that : $C=c \mathcal{E}$. Assume also that all ducts are cylindrical, implying : $\mathcal{A}^{\prime}=0$. The eigenvalue problem (12) then reduces to :

$$
\left\{\begin{array}{l}
\phi_{i_{1} i_{2}} \in C^{2}([0,1])\left(e_{i_{1} i_{2}}=0 \Rightarrow \phi_{i_{1} i_{2}}=0\right) \forall i_{1}, i_{2} \in \mathcal{I} \\
\mathcal{L}^{(-2)} \star \Phi^{\prime \prime}(\xi)=-\frac{\omega^{2}}{c^{2}} \Phi(\xi), \forall \xi \in[0,1] \\
\exists \psi \in \mathbb{R}^{N+1}: \Phi(0)=\left(\psi z^{T}\right) \star \mathcal{E} \\
{\left[\mathcal{L}^{(-1)} \star \mathcal{A} \star \Phi^{\prime}(0)\right] z=0} \\
\Phi^{T}(\xi)=\Phi(1-\xi), \forall \xi \in[0,1]
\end{array}\right.
$$

\subsection{Solution of the eigenvalue problem}

Due to the form of the matrix differential equation in (13), setting as usual $k=\frac{\omega}{c}$ for the wave number, a trigonometric matrix ansatz: $\Phi(\xi)=A \cos (k \mathcal{L} \xi)+B \sin (k \mathcal{L} \xi)$ can be tried. Using the Hadamard calculus above, the boundary condition and the symmetry property for $\Phi$, the matrix solution of problem (13) is then shown to be :

$$
\Phi(\xi)=\cos (k \mathcal{L} \xi) \star \Phi(0)+\frac{1}{k} \mathcal{L}^{(-1)} \star \sin (k \mathcal{L} \xi) \star \Phi^{\prime}(0)
$$

that can be checked by simple substitution into equation (13). It gives a closed-form expression of the eigenvector $\Phi(\xi)$ corresponding to an eigenvalue $k$. Notice its close formal resemblance with the solution of an initial value problem for the usual scalar wave equation. This is due to the rescaling and matrix encoding of the variables through graph theory in 4.2 . Remark also that the matrix initial value $\Phi(0)$ includes the values of $\varphi$ at the internal nodes, which are unknown until now ( $k$ is unknown) and imposed by the conditions at the junctions. Expression (14) is a key point as it gives the solution of a somewhat complex eigenvalue problem in a comprehensible form : thanks to the symmetry of $\Phi$, one has, for $\xi=1: \Phi(0)^{T}=\Phi(1)$. From equation (14), one gets : $\Phi(0)^{T}=\Phi(1)=\cos (k \mathcal{L}) \star \Phi(0)+\frac{1}{k} \mathcal{L}^{(-1)} \star \sin (k \mathcal{L}) \star \Phi^{\prime}(0)$. Solving this for $\Phi^{\prime}(0)$, substituting into the boundary equation (fourth line) and using the third line of (13), one gets after some simple algebra the generalized eigenvalue problem :

$$
\begin{aligned}
0 & =\left(\mathcal{A} \star(\sin (k \mathcal{L}))^{-1} \star \Phi(0)^{T}\right) z-\left(\mathcal{A} \star(\sin (k \mathcal{L}))^{-1} \star \cos (k \mathcal{L}) \star \Phi(0)\right) z \\
& =\left(\mathcal{A} \star(\sin (k \mathcal{L}))^{-1}\right) \psi-\operatorname{diag}\left[\left(\mathcal{A} \star(\sin (k \mathcal{L}))^{-1} \star \cos (k \mathcal{L})\right) z\right] \psi
\end{aligned}
$$

provided $\sin k L_{j} \neq 0 \forall j \in \mathcal{J}$. Observe that when $\sin k L_{j}=0$, one gets the natural frequencies of the individual edge $j$. Now, in view of (15) (see [19]), define the characteristic matrix :

$$
\mathcal{M}(k)=\mathcal{A} \star(\sin (k \mathcal{L}))^{-1}-\operatorname{diag}\left[\left(\mathcal{A} \star(\sin (k \mathcal{L}))^{-1} \star \cos (k \mathcal{L})\right) z\right]
$$

with $c, \mathcal{L}, \mathcal{A}, \mathcal{E}$ the given physical and geometric parameters. The consequence of (15) is that a nontrivial $\psi$ must belong to the kernel of $\mathcal{M}(k)$ and the eigenvalues then are the solution of

$$
\operatorname{det} \mathcal{M}(k)=0
$$

When all lengths of individual edges of the graph are equal to a given length $L$, the expression (16) greatly simplifies and the nonlinear problem (17) can be shown, provided $\sin (k L) \neq 0$, to reduce to the algebraic eigenvalue problem $[8,19]$ :

$$
\mathcal{A} \psi=\cos (k L) \operatorname{diag}(\mathcal{A} z) \psi
$$


i.e. defining the matrix $Z:=(\operatorname{diag}(\mathcal{A} z))^{-1} \mathcal{A}$ and $\mu=\cos (k L)$, to the eigenvalue problem :

$$
Z \psi=\mu \psi
$$

A consequence is that the values of the eigenvectors at all nodes, including the interior ones, i.e. $\Phi(0)$, are readily computed at once (through the third line of (13)). Therefore, through the above sequence of mathematical transformations, the complete set of natural frequencies and values of eigenmodes at the vertices of the graph for the wind instrument is computed : for each eigenvalue $\mu$ of $Z$, an infinite sequence $k L=\arccos (\mu)+$ $2 m \pi, m \in \mathbb{Z}$ is obtained, hence the infinite sequence of natural frequencies of the instrument. Albeit slightly mathematically involved, the method nevertheless is quite easy to implement on standard numerical softwares.

\subsection{Boundary conditions}

Remember that in this work only simplified boundary conditions are considered : the pressure (resp. volume velocity) is assumed to vanish when a termination is open (resp. closed). Let us indicate how this translates within the present approach. As both are expressed through the velocity potential, consider this one only. Assume for example that in figure 2, the vertex $V_{1}$ is open : $\varphi_{1}(0, t)=0$, with edge $E_{1}$ oriented left to right. The corresponding first line of $\Phi(0)$ (resp. the first column of $\Phi^{T}(0)$ ) vanishes identically, as for this simple vertex $\varphi_{1}(0, t)=\phi_{12}(0)$ is the only generically nonzero element in this first line (this can be checked on the simple illustrating example of $\Phi$, Eq. (11)). Due to the definition of $\psi$ in (13), its first element $\psi_{1}$ is exactly $\phi_{12}(0)$. As it vanishes, the first scalar equation in (15) becomes trivial $(0=0)$ : the dimension of the matrix system (15) is thus one less. Expliciting (15) shows that it merely amounts to cancelling the first line and row of $\mathcal{M}(k)$. The same argument applies when several external vertices are open. When an external vertex is closed, there is no condition on $\varphi_{1}$ (only on $\partial_{x_{1}} \varphi_{1}$, i.e. a Neumann condition) thus the corresponding first line and column are kept untouched. As a conclusion, for computing natural frequencies in the sequel, an open vertex is accounted for by cancelling the corresponding line and column in $\mathcal{M}(k)$ and the eigenvalues are computed from the roots of the reduced matrix determinant. Notice that the labellings in figure 2 have been chosen so that the right end corresponds to line and column of $\mathcal{M}(k)$ with index $N+1$. Whenever toneholes are considered, the lowest one corresponds to the line and row with second largest index and so on : opening successive toneholes implies cancelling more and more lines and columns from the $N^{\text {th }}$ ones.

\section{Application 1 : piecewise cylindrical resonators without toneholes}

The symbolic computations in the present and following sections could hardly have been done with the transmission lines approach, except in the most elementary situations. No analogous ones seem to have appeared in the literature. First results have been presented in [21] and numerical computations using the following developments have been conducted in [22], all for a low number $(\leq 4)$ of cylinders with equal lengths. 


\subsection{Graph description}

The graph of a piecewise cylindrical resonator without toneholes is a mere sequence of $N$ edges with maximum vertex degree two, i.e. each internal vertex has exactly two neighbouring vertices. The consequence is that the $(N+1) \times(N+1)$-matrices $\mathcal{E}, \mathcal{A}, \mathcal{L}$ are tridiagonal with vanishing main diagonal and $\mathcal{D}$ is $(N+1) \times N$ bidiagonal. Thus, instead of writing them explicitely, a special notation is used for saving space : $\operatorname{diag}(\mathrm{v}, j)$ stands for a matrix with $j$ th diagonal $(j \in \mathbb{Z})$ given by the vector $\mathrm{v}$, and zeroes elsewhere. When $j=0$, it is the main diagonal and when $j<0$ (resp. $j>0$ ), it is the $j$ th diagonal under (resp. above) the main diagonal. Such matrices can be added provided convenient dimensions are given. Set Q (resp. A, resp. L), the $N$-vector with all elements equal to one (resp. the cross-sections areas $a_{i}$, resp. the lengths $L_{i}$, of the edges). Then, a resonator made of $N$ concatenated cylinders with lengths $L_{i}$ and cross-sections $a_{i}$ is described by the matrices :

$$
\begin{gathered}
\mathcal{E}=\operatorname{diag}(\mathrm{Q},-1)+\operatorname{diag}(\mathrm{Q}, 1), \mathcal{D}=\operatorname{diag}(-\mathrm{Q}, 0)+\operatorname{diag}(\mathrm{Q},-1), \\
\mathcal{A}=\operatorname{diag}(\mathrm{A},-1)+\operatorname{diag}(\mathrm{A}, 1), \mathcal{L}=\operatorname{diag}(\mathrm{L},-1)+\operatorname{diag}(\mathrm{L}, 1)
\end{gathered}
$$

Its characteristic matrix $\mathcal{M}(k)$ writes :

$$
\mathcal{M}(k)=\operatorname{diag}(\alpha, \boldsymbol{\theta})+\operatorname{diag}(\beta,-1)+\operatorname{diag}(\beta, 1)
$$

with $\alpha=\left(\alpha_{i}\right)_{i=1, \ldots, N+1}, \beta=\left(\beta_{i}\right)_{i=1, \ldots, N}$ and : $\alpha_{1}=-a_{1} \frac{\lambda_{1}}{\gamma_{1}}, \alpha_{N+1}=-a_{N} \frac{\lambda_{N}}{\gamma_{N}}, \alpha_{i}=$ $-\left(a_{i-1} \frac{\lambda_{i-1}}{\gamma_{i-1}}+a_{i} \frac{\lambda_{i}}{\gamma_{i}}\right), i=2, \ldots, N, \beta_{i}=\frac{a_{i}}{\gamma_{i}}, i=1, \ldots, N$ and $\lambda_{i}=\cos \left(k L_{i}\right), \gamma_{i}=\sin \left(k L_{i}\right)$. The fact that $\mathcal{M}(k)$ is tridiagonal symmetric allows for fast computations. Needless to say, although the matrix $\mathcal{M}(k)$ is easily constructed, solving the characteristic equation $\operatorname{det} \mathcal{M}(k)=0$ explicitely by brute force is hopeless as its complexity grows very quickly with the number of cylinders. As seen at the end of section 4.3, the special case when lengths $L_{i}$ are all equal to the same $L$ reduces the search for natural frequencies to an algebraic eigenvalue problem, for which efficient solvers exist. Under this assumption, let $\lambda=\lambda_{i} \equiv \cos (k L), \gamma=\gamma_{i} \equiv \sin (k L)$. Then $\mathcal{M}(k)$ writes :

$$
\mathcal{M}(k)=\frac{1}{\gamma}(\operatorname{diag}(\alpha, 0)+\operatorname{diag}(\beta,-1)+\operatorname{diag}(\beta, 1)
$$

where the definitions of $\alpha_{i}, \beta_{i}$ have been changed to : $\alpha_{1}=-a_{1} \lambda, \alpha_{N+1}=-a_{N} \lambda, \alpha_{i}=$ $-\left(a_{i-1}+a_{i}\right) \lambda, i=2, \ldots, N, \beta_{i}=a_{i}$. Even for resonators with complex geometries having parts with different lengths, a sufficiently fine discretization into equal length cylinders allows the above simplification. This is at the price of increasing the dimension of $\mathcal{M}(k)$. But the resulting problem is easily amenable to a numerical solution and even allows for certain formal computations, using a computer algebra system, as it will be seen below. Notice also that the graph laplacian matrix or Kirchoff matrix, obtained when approximating the 1D laplacian by finite differences on a regular mesh with vanishing Neumann boundary conditions, is the same as above when all $a_{i}$ 's are equal. This corresponds to a mere discretized cylinder, in a coherent way.

\subsection{Closed-open piecewise cylindrical resonators, $N \in \mathbb{N}$}

Consider a piecewise cylindrical resonator for which all lengths of individual elements are equal to a unique $L$. No special relationship between the cross sections $a_{i}$ is assumed for the time being. Consider, as in [12], the case were one end, say the left, 
is closed and the other end is open. Thus the potential vanishes at the latter end. As a consequence (see section 4.4) the last row and column of $\mathcal{M}(k)$ are discarded when solving the algebraic eigenvalue problem (see section 4.4). This leads to the following characteristic matrix, still denoted $\mathcal{M}(k)$ :

$$
\mathcal{M}(k)=\frac{1}{\gamma}\left(\begin{array}{rrrrr}
\alpha_{1} \lambda & \beta_{1} & 0 & \cdots & 0 \\
\beta_{1} & \alpha_{2} \lambda & \beta_{2} & \cdots & 0 \\
0 & \beta_{2} & \alpha_{3} \lambda & \ddots & 0 \\
\vdots & \ddots & \ddots & \ddots & \vdots \\
0 & 0 & \cdots & \beta_{N-1} & \alpha_{N} \lambda
\end{array}\right)
$$

with this time : $\alpha_{1}=-a_{1}, \alpha_{i}=-\left(a_{i-1}+a_{i}\right), i=2, \ldots, N, \beta_{i}=a_{i}$. Thanks to its tridiagonal symmetric structure, it is straightforward to show that its determinant $p_{N}(\lambda)$ can be computed recursively, discarding $\gamma \neq 0$, as :

$$
\left\{\begin{array}{l}
p_{0}(\lambda)=1 \\
p_{1}(\lambda)=\alpha_{1} \lambda \\
p_{n}(\lambda)=\alpha_{n} \lambda p_{n-1}(\lambda)-\beta_{n-1}^{2} p_{n-2}(\lambda), n=2, \ldots,
\end{array}\right.
$$

This three terms recursion (23) is computationally very efficient. Moreover, it suggests an interpretation of the family $\left\{p_{n}(\lambda)\right\}$ in terms of orthogonal polynomials [27] but this remains to be studied in full generality. Such a recursion reminds the one found in [12] for the diameters. For the time being, one restricts, as a matter of example, to the special class of [12].

The special progression $a_{n}=\frac{n(n+1)}{2} a_{1} \quad$ A whole class of closed-open resonators with discontinuities has been shown [12] to have harmonically related natural frequencies : piecewise cylinders such that the sequence of cross-section areas is in the progression $a_{n}=\frac{n(n+1)}{2} a_{1}$. It was conjectured that they should be the only ones with this property. But within the present approach, a wider class will be evidenced below. One can show that $p_{n}(\lambda)$ is a particular case of Jacobi polynomial. More precisely, the following identity is true : $p_{n}(\lambda)=(-1)^{n} g(n) G_{n}(\lambda)$ where $G_{n}(\lambda)$ is the $n^{\text {th }}$ ultraspherical polynomial [27] : $G(n, a, \lambda)$ with $a=1 ; g(n)$ is a factor that depends only on $n$, the degree of the polynomial i.e. the number of cylinders constituting the resonator, and modifies only the normalization of the family. Hence the family $\left\{p_{n}(\lambda)\right\}, n=1, \ldots$ is also a polynomial family, that is orthogonal on the interval $[-1,+1]$ with respect to the weight function $w(\lambda)=\sqrt{1-\lambda^{2}}$. In this particular case, the zeroes, $\zeta_{l, n}$, of each $p_{n}(\lambda)$ are those of $G_{n}(\lambda): \zeta_{l, n}=\cos \left(\frac{l \pi}{n+1}\right), l=1, \ldots, n$. Thus the $(n+1)^{t h}$ multiple of the argument is lacking in the series. As these zeroes furnish the natural frequencies of the resonator, one important property evidenced in [12] is thus recovered : for $n$ concatenated such cylinders, the partial, which is harmonic, with rank $n+1$ is missing in the series. Also, for an odd number of cylinders, the natural frequencies of the basic element are also present ( $\lambda$ divides $p_{n}$, see also item 1 in section 4.3). In our opinion, this class seems to play a special role in the whole set of resonators with harmonically related natural frequencies that have been evidenced with the present method [22] but we cannot be more precise at this stage.

Resonators with harmonically related natural frequencies $\quad$ : In [22], it was mentionned that the natural frequencies of a piecewise cylindrical resonator, with equal length $L$, 
are harmonically related if and only if $\frac{k L}{\pi}$ is a rational number. Let us explain this fact : the natural frequencies are the roots of $p_{n}(\lambda), \lambda=\cos (k L)$. It can be shown, thanks to the recursion (23), that only $\lambda^{2}$ comes into play, except when $n$ is odd, where $\lambda=0$, i.e. $k L=\pi / 2(\bmod \pi)$, is the supplementary solution. Thus the roots come by pairs : if $k L$ brings a solution, $\pi-k L$ brings one too. Thus one can fix only $n / 2$ roots for $p_{n}$ as the other $n / 2$ ones are also fixed by this property. Denoting $k_{1}$ (resp. $k_{n}$ ) the wave number for the first (resp. $n^{t h}$ ) natural frequency, one has necessarily $\frac{f_{n}}{f_{1}}=\frac{k_{n}}{k_{1}} \in \mathbb{N}$, in order for $f_{n}$ to be harmonically related to $f_{1}$. Solving $\cos ^{2}\left(k_{n} L\right)=\cos ^{2}\left(k_{1} L\right)$ results in $k_{n} L=k_{1} L+2 l \pi$ or $k_{n} L=\pi-k_{1} L+2 j \pi, j, l \in \mathbb{N}$, which implies that $\frac{k_{1} L}{\pi}$ must be a rational number. Observe that the chosen values for the $n / 2$ different $k_{i} L$ are obviously necessary but unsufficient data to determine the $n$ cross-sections of such a resonator. This answers negatively to the above-mentionned conjecture made in [12]. Actually, it is known that giving only the sequence of eigenvalues of a Sturm-Liouville equation as Eq. (1), on the unit interval with vanishing boundary conditions, is unsufficient to reconstruct the unknown coefficient of this equation [28] : in the present situation, this means that specifying only that the natural frequencies are harmonically related cannot determine uniquely the shape of the duct. For more theoretical considerations about this question of spectral geometry, see [24] or a quick summary in the appendix of [29].

\subsection{Closed-open piecewise cylindrical resonator, $N=2$}

As a first simple illustration of how the method proceeds one summarizes an example given in [21], while considering here the closed-open case only. The full characteristic matrix writes :

$$
\mathcal{M}(k)=\left(\begin{array}{ccc}
-a_{1} \frac{\lambda_{1}}{\gamma_{1}} & \frac{a_{1}}{\gamma_{1}} & 0 \\
\frac{a_{1}}{\gamma_{1}} & -\left(a_{1} \frac{\lambda_{1}}{\gamma_{1}}+a_{2} \frac{\lambda_{2}}{\gamma_{2}}\right) & \frac{a_{2}}{\gamma_{2}} \\
0 & \frac{a_{2}}{\gamma_{2}} & -a_{2} \frac{\lambda_{2}}{\gamma_{2}}
\end{array}\right)
$$

The natural frequencies are obtained as those values that make vanish the determinant of the first $2 \times 2$ sub-matrix, which is : $-a_{1}+a_{2} \frac{\lambda_{1}}{\gamma_{1}} \frac{\lambda_{2}}{\gamma_{2}}$. They are the solutions of the transcendental equation :

$$
\tan \left(k L_{1}\right) \tan \left(k L_{2}\right)=\frac{a_{2}}{a_{1}}
$$

which is recognized to be Eq. (7.19), p. 259 in [4]. Exchanging closed and open ends, the natural frequencies are the solution of $: \tan \left(k L_{1}\right) \tan \left(k L_{2}\right)=\frac{a_{1}}{a_{2}}$. Notice the symmetry between both equations. When $L_{1}=L_{2}=L$, one gets : $\tan ^{2}(k L)=\frac{a_{2}}{a_{1}}$ for the closed-open case and : $\tan ^{2}(k L)=\frac{a_{1}}{a_{2}}$ in the open-closed case. This means that they are dual stepped-cones of each other : one is convergent, this other is divergent. Thus two geometries have the same series of harmonically related natural frequencies.

\subsection{Closed-open piecewise cylindrical resonator, $N=3$}

Here again the exposition in [21] is summarized and limited to the closed-open situation. The full characteristic matrix is :

$$
\mathcal{M}(k)=\left(\begin{array}{cccc}
-a_{1} \frac{\lambda_{1}}{\gamma_{1}} & \frac{a_{1}}{\gamma_{1}} & 0 & 0 \\
\frac{a_{1}}{\gamma_{1}} & -\left(a_{1} \frac{\lambda_{1}}{\gamma_{1}}+a_{2} \frac{\lambda_{2}}{\gamma_{2}}\right) & \frac{a_{2}}{\gamma_{2}} & 0 \\
0 & \frac{a_{2}}{\gamma_{2}} & -\left(a_{2} \frac{\lambda_{2}}{\gamma_{2}}+a_{3} \frac{\lambda_{3}}{\gamma_{3}}\right) & \frac{a_{3}}{\gamma_{3}} \\
0 & 0 & \frac{a_{3}}{\gamma_{3}} & -a_{3} \frac{\lambda_{3}}{\gamma_{3}}
\end{array}\right)
$$


When $L_{1}=L_{2}=L_{3}=L$, the natural frequencies for the closed-open situation are found through the recursion (23) to satisfy $p_{3}(\lambda)=0$ (see [22] for more details). From this, the closed-form expression for $a_{3}$ is :

$$
a_{3}=\frac{a_{2}\left(a_{1}+a_{2}\right)\left(1-\lambda^{2}\right)}{\left(a_{1}+a_{2}\right) \lambda^{2}-a_{1}}
$$

$a_{1}$ can be taken as unit and $a_{2}$ as a free parameter. One must have $a_{2}>\frac{a_{1}\left(1-\lambda^{2}\right)}{\lambda^{2}}$, in order to have a positive, physically relevant, solution for $a_{3}$. Any resonator with three cylinders satisfying these relations will then have harmonically related frequencies, provided one fixes $a_{1}$ and chooses $k$ such that $\frac{k L}{\pi}$ is a rational number. Each value of the latter leads to a particular series. Contrary to the case $N=2$, fixing $a_{1}$ resp. $k$ to a given value, one is left with one equation and two unknowns thus there is an infinite family of solutions. But, whereas for $N=2$ the two possible solutions can be derived using the classical approach, it would have been much more difficult to obtain the answer for $N=3$ (see [12]). The usefulness of formulae such as (27) that can be obtained for any $N$ is that they give an explicit and constructive form to this non uniqueness property hence can be useful for design (see [23] e.g.). More but still preliminary computations founded on the present theoretical approach have been conducted in [22] for a low number $(N \leq 4)$ of cylinders.

\section{Application 2 : resonators with toneholes}

After the rather simple resonators above, the purpose of this section is to show that the method easily furnishes closed-form expressions for instruments with toneholes. These expressions can then be used for numerical computations. Contrary to previous studies $[10,5]$, no regularity is assumed for the repartition and sizes of the holes. No supplementary approximations are done neither within the present framework. Two problems are touched upon : the direct problem consists in computing the natural frequencies for a given geometry of the instrument. The inverse problem proceeds the reverse way : fixing the series of natural frequencies, one looks for a geometry of the instrument. The purpose here again is merely to illustrate the potential of the method. In particular, one assertion of A.H. Benade [2], about the effect of opening toneholes beyond the first two or three ones is studied quantitatively on one example.

\subsection{Woodwind with one tonehole}

This oversimplified wind instrument has been deeply studied within the classical approach [10, 5, 13, 4]. First numerical results within the present approach are in [21]. The graph of a one tonehole woodwind is star-shaped with three edges all connected at one vertex (set $h=1$ in figure 2). Choosing the orientation of the three edges for which the origin is at the only multiple vertex $V_{2}$, the relevant matrices are :

$$
\mathcal{D}=\left(\begin{array}{ccc}
1 & 0 & 0 \\
-1 & -1 & -1 \\
0 & 0 & 1 \\
0 & 1 & 0
\end{array}\right), \mathcal{E}=\left(\begin{array}{llll}
0 & 1 & 0 & 0 \\
1 & 0 & 1 & 1 \\
0 & 1 & 0 & 0 \\
0 & 1 & 0 & 0
\end{array}\right)
$$


with $\mathcal{A}, \mathcal{L}$ having the same pattern as $\mathcal{E}$ and the characteristic matrix is :

$$
\mathcal{M}(k)=\left(\begin{array}{cccc}
-\frac{a_{1} \lambda_{1}}{\gamma_{1}} & \frac{a_{1}}{\gamma_{1}} & 0 & 0 \\
\frac{a_{1}}{\gamma_{1}} & -\sum_{i=1}^{3} \frac{a_{i} \lambda_{i}}{\gamma_{i}} & \frac{a_{3}}{\gamma_{3}} & \frac{a_{2}}{\gamma_{2}} \\
0 & \frac{a_{3}}{\gamma_{3}} & -\frac{a_{3} \lambda_{3}}{\gamma_{3}} & 0 \\
0 & \frac{a_{2}}{\gamma_{2}} & 0 & -\frac{a_{2} \lambda_{2}}{\gamma_{2}}
\end{array}\right)
$$

Writing the equations satisfied by the natural frequencies is not more complex than for a resonator without toneholes : the formal computations remain the same, only the pattern of the matrices $\mathcal{E}, \mathcal{D}, \mathcal{A}, \mathcal{L}$ changes and this makes a big difference with the transmission lines method. Consider for example the situation with the left end closed, the right end open and the tonehole closed. The corresponding equation for natural frequencies is :

$$
a_{1} \tan \left(k L_{1}\right)+a_{3} \tan \left(k L_{3}\right)-\frac{a_{2}}{\tan \left(k L_{2}\right)}=0
$$

The second term of the left hand side gives the exact perturbation term, within the plane wave approximation, due to the closed tonehole -compare e.g. with the treatment in [4], pp 302-319. It allows for a precise study of the effect of the tonehole geometry on the natural frequencies of this elementary woodwind (see section 6.3 below). Moreover, letting $a_{3}$ go to zero amounts to suppressing the tonehole. It leads to : $a_{1} \tan \left(k L_{1}\right)=$ $\frac{a_{2}}{\tan \left(k L_{2}\right)}$ which is nothing else than equation (25), in a coherent way as this limiting situation is just a piecewise cylindrical resonator made of two cylinders as in section 5.3. Equation (30) can be used to solve the direct problem when the tonehole is closed. Although highly nonlinear, in that case it is a function of one variable, $k$, the solutions of which are to be found. Firstly, the singularities are those of the tangent and cotangent functions. Zeroes are localized in each interval in between and a standard numerical method allows to compute them.

Similarly the equation giving natural frequencies for the open tonehole is obtained by computing the determinant of the first $2 \times 2$ sub-matrix of the characteristic matrix (i.e. cancelling the last two lines and columns in (29)), which gives :

$$
a_{1} \tan \left(k L_{1}\right)-\frac{a_{2}}{\tan \left(k L_{2}\right)}-\frac{a_{3}}{\tan \left(k L_{3}\right)}=0
$$

With the same method as above, the zeroes can be found outside the singularities of the left hand side.

Using these results, a typical inverse problem also can be solved with no more approximation along the following lines. Assume one wants e.g. to design the instrument such that the wavenumbers when opening and closing the tonehole correspond to a given musical interval (a third, a fifth or whatever else). Denoting $k^{c}$ (resp. $k^{o}$ ) the wave number in the closed (resp. open) tonehole situation, this imposes : $\frac{k^{c}}{k^{c}}=\mu$, with $\mu \in \mathbb{R}$, depending on the chosen interval. From ergonomy or other considerations, assume also that the lengths $L_{i}, i=1,2,3$ are fixed. Consider the system of two equations (30), (31) in that situation :

$$
\left\{\begin{array}{l}
a_{1} \tan \left(k^{c} L_{1}\right)+a_{3} \tan \left(k^{c} L_{3}\right)-\frac{a_{2}}{\tan \left(k^{c} L_{2}\right)}=0 \\
a_{1} \tan \left(k^{o} L_{1}\right)-\frac{a_{2}}{\tan \left(k^{o} L_{2}\right)}-\frac{a_{3}}{\tan \left(k^{o} L_{3}\right)}=0
\end{array}\right.
$$

Fixing $a_{1}$ as unit, $a_{2}$ and $a_{3}$ are obtained as the solutions of this simple linear system. For other inverse problems involving lengths $L_{i}$, things are not that simple and ask for more work. This toy problem thus only gives an illustration of what can be done but opens the way towards more realistic studies. 


\subsection{Woodwind with two toneholes}

Consider, as a more complex example within the present approach, a woodwind with two toneholes. It is described by its graph, setting $h=2$ in figure 2 , and its characteristic matrix is :

$$
\mathcal{M}(k)=\left(\begin{array}{cccccc}
-\frac{a_{1} \lambda_{1}}{\gamma_{1}} & \frac{a_{1}}{\gamma_{1}} & 0 & 0 & 0 & 0 \\
\frac{a_{1}}{\gamma_{1}} & -\sum_{i=1,2,4} \frac{a_{i} \lambda_{i}}{\gamma_{i}} & \frac{a_{2}}{\gamma_{2}} & \frac{a_{4}}{\gamma_{4}} & 0 & 0 \\
0 & \frac{a_{2}}{\gamma_{2}} & -\sum_{i=2,3,5} \frac{a_{i} \lambda_{i}}{\gamma_{i}} & 0 & \frac{a_{5}}{\gamma_{5}} & \frac{a_{3}}{\gamma_{3}} \\
0 & \frac{a_{4}}{\gamma_{4}} & 0 & -\frac{a_{4} \lambda_{4}}{\gamma_{4}} & 0 & 0 \\
0 & 0 & \frac{a_{5}}{\gamma_{5}} & 0 & -\frac{a_{5} \lambda_{5}}{\gamma_{5}} & 0 \\
0 & 0 & \frac{a_{3}}{\gamma_{3}} & 0 & 0 & -\frac{a_{3} \lambda_{3}}{\gamma_{3}}
\end{array}\right)
$$

Notice that this describes the most general situation, not limited to uniform sizings. It does not seem to have been studied before in this generality. Here again assume the left end closed and the right end open. Considering that both toneholes are closed implies that one cancels only the last column and line in the characteristic matrix (33) (see section 4.4). The natural frequencies are those values of $k$ that make vanish the determinant of the resulting $5 \times 5$ matrix. As a first check of correctness, letting $a_{4}$ and $a_{5}$ go to zero amounts to consider the same instrument without toneholes. It is straightforward to verify that this leads to a characteristic matrix that is exactly the principal $3 \times 3$ submatrix of (26) for $N=3$. This is coherent, as the limiting case is nothing else than a cylinder made with three elementary parts. Now, for studying the effect of opening the lowest hole solely (vertex $V_{5}$, see figure 2 with $h=2$ ), the relevant characteristic matrix is obtained by cancelling moreover the fifth line and column from (33). Setting $g_{i}=\frac{a_{i}}{\tan \left(k L_{i}\right)}$, its determinant gives the equation to be satisfied for the natural frequencies :

$$
a_{1}^{2} g_{4}\left(g_{2}+g_{3}+g_{5}\right)-g_{1}\left(g_{4}\left(g_{2}\left(g_{3}+g_{5}\right)-a_{2}^{2}\right)+a_{4}^{2}\left(g_{2}+g_{3}+g_{5}\right)\right)=0
$$

When the second hole (vertex $V_{4}$ ) solely is open, the characteristic matrix is obtained similarly by cancelling the fourth and sixth lines and columns from (33), giving the equation for the natural frequencies :

$$
a_{1}^{2}\left(a_{5}^{2}-g_{5}\left(g_{2}+g_{3}\right)\right)-g_{1}\left(a_{2}^{2} g_{5}+g_{2}\left(a_{5}^{2}-g_{5}\left(g_{3}+g_{4}\right)\right)+g_{4}\left(a_{5}^{2}-g_{3} g_{5}\right)\right)=0
$$

while opening both toneholes is obtained by keeping only the principal $3 \times 3$ submatrix of (33), leading to the natural frequencies equation :

$$
a_{1}^{2}\left(g_{2}+g_{3}+g_{5}\right)+g_{1}\left(a_{2}^{2}-g_{2}\left(g_{3}+g_{4}+g_{5}\right)-g_{4}\left(g_{3}+g_{5}\right)\right)=0
$$

and allows to study the effect of the two open toneholes on the natural frequency of the upper part of the duct, made of the cylinder with length $L_{1}$ alone. For each of the three equations (34), (35), (36), the method suggested in section 6.1 can be applied for computing the natural frequencies : in a first step, localize them in intervals bounded by singularities of the cotangent functions $g_{i}$. In a second step, compute those in a given interval thanks to a standard numerical method. The lowest one is the fundamental frequency for the given fingering. This way, the effect of opening any tonehole on the natural frequencies of the instrument can be quantified. We do not go into numerical computations, leaving them to a more realistic example in the following section. 


\subsection{Woodwind with three toneholes}

According to A.H. Benade [10, 2], the frequency and tone quality of a given played note are almost wholly independent of the size and position of the open holes beyond the first two or three. This observation motivated the use of an equivalent uniform transmission lines. This assertion will be checked on a simple example within the presented approach. Firstly, for an instrument with three toneholes, set $h=3$ in figure 2 and build the full $8 \times 8$ characteristic matrix (16). Then, assuming that the instrument belongs to the family of flutes, both ends of the main duct are open : this is tantamount to cancelling the first and last columns and lines of this matrix (see section 4.4). When the three toneholes are open, corresponding lines and columns are cancelled too. The resulting $3 \times 3$ characteristic matrix :

$$
\mathcal{M}_{1}(k)=\left(\begin{array}{ccc}
-\sum_{i=1,2,5} \frac{a_{i} \lambda_{i}}{\gamma_{i}} & \frac{a_{2}}{\gamma_{2}} & 0 \\
\frac{a_{2}}{\gamma_{2}} & -\sum_{i=2,3,6} \frac{a_{i} \lambda_{i}}{\gamma_{i}} & \frac{a_{3}}{\gamma_{3}} \\
0 & \frac{a_{3}}{\gamma_{3}} & -\sum_{i=3,4,7} \frac{a_{i} \lambda_{i}}{\gamma_{i}}
\end{array}\right)
$$

is the basic object for the present study. The focus will be on the note pitch, corresponding to the first zero of $\operatorname{det} \mathcal{M}_{1}(k)$. The bottom part of a flute in $C$ has been chosen for illustration : it is derived from a renaissance tenor flute [30] of which only the three bottom toneholes have been kept. The dimensions have been made uniform and rounded : surely this instrument is not just in tune but one is interested here in quantifying the effect of second and third tonehole dimensions and positions only. The chosen dimensions of the main duct, having diameter $17 \mathrm{~mm}$, appear in table 1 . When all

\begin{tabular}{|c|c|c|c|c|}
\hline Note & C & D & E & F \\
\hline Tube length $(\mathrm{mm})$ & 631 & 532 & 494 & 453 \\
\hline
\end{tabular}

Table 1: Dimensions of the flute model with 3 toneholes

toneholes are open, the played note is $F$, with frequency $352 \mathrm{~Hz}$ in a natural scale with just intonation and $A$ at $440 H z$. The sound velocity is $c=343 \mathrm{~m} / \mathrm{s}$ and the nominal dimensions for computations appear in table 2. Referring to figure $2, L_{i}$ is the length of the $i^{t h}$ edge and $D_{i}$ its diameter, $a_{i}=\pi D_{i}^{2} / 4$. Naturally, the theoretical fundamental frequency for the note $F$ differs from the following results as all geometrical parameters come into play, especially the relative dimensions of the main duct and toneholes.

The computed results appear in tables 3, 4, 5, with nominal values in the third

\begin{tabular}{|c|c|c|c|c|c|c|c|c|c|c|c|c|c|}
\hline$L_{1}$ & $L_{2}$ & $L_{3}$ & $L_{4}$ & $L_{5}$ & $L_{6}$ & $L_{7}$ & $D_{1}$ & $D_{2}$ & $D_{3}$ & $D_{4}$ & $D_{5}$ & $D_{6}$ & $D_{7}$ \\
\hline 453 & 41 & 38 & 99 & 6 & 6 & 6 & 17 & 17 & 17 & 17 & 6 & 6 & 6 \\
\hline
\end{tabular}

Table 2: Dimensions for the graph of the flute model, in mm

column of each table. One parameter at a time was varied around its nominal value. Varying the position of the second (resp. third) tonehole is done through varying the length $L_{2}$ (resp. $L_{3}$ ) on figure 2, while keeping the nominal length $L_{2}+L_{3}=79 \mathrm{~mm}$ (resp. $L_{3}+L_{4}=137 \mathrm{~mm}$ ) constant. To complement this, table 6 shows the maximum relative variation in frequency : for each parameter par, it gives $\Delta_{p a r}=\max \frac{\left|f-f_{\text {nom }}\right|}{f_{\text {nom }}}$ in cents and in percentage of the frequency, $f_{\text {nom }}=355.7 \mathrm{~Hz}$, obtained for nominal parameters. The influence of the third tonehole parameters is significantly less than for the second tonehole, as one can expect. The maximum relative frequency variation 
for the third tonehole is less than 8 cents $(0.5 \%)$, for all parameters $L_{7}, D_{7}, L_{3}$. It is well below the difference limen for perception of pure tones [31], chap.2. As for the second tonehole, the position $\left(L_{2}\right)$ has no significant influence. The tonehole length effect $\left(L_{6}\right)$ is slightly above the low limit of perception. Only the diameter $\left(D_{6}\right)$ acts significantly on the natural frequency but for a rather important variation $(66 \%)$ of its nominal value. Nevertheless, it remains close to the difference limen for perception. One can thus conclude that the claim at the beginning of this section is clearly true on this example, as far as the note pitch is concerned. Another way to study the influence of the second (resp. third) tonehole is to compare the frequencies when it is open and then closed, while keeping the first and the third (resp. second) ones open. In each case, the characteristic matrix is obtained as above by a relevant selection of lines and columns in the full $8 \times 8$ original one. Without going into the computational details, the results are the following : for the nominal dimensions, when the third tonehole only is closed, the first natural frequency is $354.69 \mathrm{~Hz}$, very close to $355.7 \mathrm{~Hz}(-5$ cents) when it is open. As for the second tonehole, when it is closed, the computed frequency is $351.4 \mathrm{~Hz}$, well below $355.7 \mathrm{~Hz}$, giving a relative frequency variation of 20 cents $(1.2 \%)$ that can be detected by ear [31], as one can expect. Notice that the directions of frequency variations found above are in accordance with the observations made in [2], p. 450, with approximate formulae. There it was said that If more exact results are needed or if the lattice is very irregular, meaningful calculations can be carried out, but they become extremely tedious and fairly subtle. With the present approach, exact expressions allow to avoid these difficulties, of course with the help of computer algebra.

\section{Conclusion}

The present work has been dedicated to the general presentation of a modelling approach for musical wind instruments, using graph theory and a convenient reformulation of the original nonstandard vector boundary value problem into a standard matrix one for the wave equation on a graph. An important consequence is an alternative method for computing the natural frequencies and an approximation of eigenmodes of these instruments, that can deal with complex assemblages of cylinders. The developments, which are to be seen as founding ones for the method, have shown that two lines of

\begin{tabular}{|c|c|c|c|c|c|}
\hline$L_{6}(\mathrm{~mm})$ & 2 & 4 & 6 & 8 & 10 \\
\hline Frequency $f(\mathrm{~Hz})$ & 358.2 & 356.7 & 355.7 & 355.1 & 354.6 \\
\hline \hline$L_{7}(\mathrm{~mm})$ & 2 & 4 & 6 & 8 & 10 \\
\hline Frequency $f(\mathrm{~Hz})$ & 356.3 & 356.0 & 355.7 & 355.6 & 355.5 \\
\hline
\end{tabular}

Table 3: Effect of the length of the second $\left(L_{6}\right)$ and third $\left(L_{7}\right)$ tonehole

\begin{tabular}{|c|c|c|c|c|c|}
\hline$D_{6}(\mathrm{~mm})$ & 2 & 4 & 6 & 8 & 10 \\
\hline Frequency $f(\mathrm{~Hz})$ & 352.2 & 354.0 & 355.7 & 357.1 & 358.0 \\
\hline \hline$D_{7}(\mathrm{~mm})$ & 2 & 4 & 6 & 8 & 10 \\
\hline Frequency $f(\mathrm{~Hz})$ & 354.9 & 355.3 & 355.7 & 356.0 & 356.3 \\
\hline
\end{tabular}

Table 4: Effect of the diameter of the second $\left(D_{6}\right)$ and third tonehole $\left(D_{7}\right)$ 


\begin{tabular}{|c|c|c|c|c|c|}
\hline$L_{2}(\mathrm{~mm})$ & 33 & 37 & 41 & 45 & 49 \\
\hline Frequency $\mathrm{f}(\mathrm{Hz})$ & 356.7 & 356.2 & 355.7 & 355.4 & 354.9 \\
\hline \hline$L_{3}(\mathrm{~mm})$ & 30 & 34 & 38 & 42 & 46 \\
\hline Frequency $\mathrm{f}(\mathrm{Hz})$ & 356.0 & 355.8 & 355.7 & 355.6 & 355.5 \\
\hline
\end{tabular}

Table 5: Effect of the position of the second $\left(L_{2}\right)$ and third $\left(L_{3}\right)$ tonehole

\begin{tabular}{|c|c|c|c||c|c|c|}
\hline & $\Delta_{L_{6}}$ & $\Delta_{D_{6}}$ & $\Delta_{L_{2}}$ & $\Delta_{L_{7}}$ & $\Delta_{D_{7}}$ & $\Delta_{L_{3}}$ \\
\hline cents & 12 & 17 & 5 & 3 & 4 & 1 \\
\hline$\%$ & 0.7 & 0.98 & 0.28 & 0.17 & 0.22 & 0.08 \\
\hline
\end{tabular}

Table 6: Maximum relative variations of frequency, in cents and in \%

investigations can now be conducted with this method: one is at the formal level as followed in this work, as it gives closed-form expressions, useful for further investigations. The other one concerns the numerical computations, as it was done in preliminary works $[21,22,23]$. As the computational complexity is relatively low, comparisons with the usual transmission lines approach should be deepened. One important result of the approach is a constructive answer given to a conjecture formulated in [12], about lattices of piecewise cylindrical sound tubes with harmonically related natural frequencies. This question was replaced in the mathematical context of inverse spectral problems [28]. One remaining question is that of characterizing the families of resonators that have harmonically related natural frequencies, while looking at a possible special role played by the progression $a_{n}=\frac{n(n+1)}{2} a_{1}$. This is currently under study after preliminary studies [22]. Getting closed-form expressions makes it possible to study anew usual questions in musical acoustics related to the natural frequencies of woodwinds. As a first example, the experimental observation that the tuning properties of a woodwind are predominantly affected by the properties of only the first two or three open toneholes [10] has been precisely quantified. Similar studies will be conducted on length corrections or the effect of different cross-fingerings on the playing frequency. A special attention will be given in the future to inverse problems, with the design in view. The excitation mechanism and related questions have to and will be accounted for in this model, as well as considering piecewise cones for the tubes. Notice eventually that the proposed approach is applicable to other acoustical studies not related to musical acoustics, in particular for 2D or 3D networks.

\section{References}

[1] H. Bouasse. Instruments à vent, I, II. Delagrave, 1929. New edition Librairie A. Blanchard, 1986.

[2] A. H. Benade. Fundamentals of musical acoustics. Dover, 1990. 2d edition.

[3] N.H. Fletcher and T. Rossing. The physics of musical instruments. Springer, 1991.

[4] A. Chaigne and J. Kergomard. Acoustique des instruments de musique. Belin, 2008. 
[5] D.H. Keefe. Woodwind air column models. J. Acoust. Soc. Am., 88(1):35-51, 1990.

[6] Benade A.H. Equivalent circuits for conical waveguides. J. Acoust. Soc. Amer. 83(5):1764-1769, 1987.

[7] R. Caussé, J. Kergomard, and X. Lurton. Input impedance of brass instruments comparison between experiment and numerical models. J. Acoustical Society of America, 75(1):241-254, 1984.

[8] G. Le Vey. Graph-based models for woodwinds. In Stockholm Music Acoustics Conference, SMAC2013, Stockholm, July 2013.

[9] R.D. Ayers, J.E. Lowell, and D. Mahgerefteh. The conical bore of in musical acoustics. Am. Jal Physics, 53(6):528-537, 1984.

[10] A.H. Benade. On the mathematical theory of woodwind finger hole. J. Acoust. Soc. Amer., 32:1591-1608, 1960.

[11] A. H. Benade and E. V. Jansson. On plane and spherical waves in horns with non uniform flare. Acustica, 31(2):79-98, 1974.

[12] J. P. Dalmont and J. Kergomard. Lattices of sound tubes with harmonically related eigenfrequencies. Acta Acustica, 2(5):421-430, 1994.

[13] V. Dubos, J. Kergomard, A. Khettabi, J. P. Dalmont, D. H. Keefe, and C. J. Nederveen. Theory of sound propagation in a duct with a branched tube using modal decomposition. Acta Acustica, 85:153-169, 1999.

[14] A. Lefebvre, G. P. Scavone, and J. Kergomard. External toneholes interaction in woodwind instruments. preprint, http://arXiv.org/pdf/1207.5490 or http://hal.archives-ouvertes.fr/hal-00720132, 2012.

[15] A. H. Benade. On woodwind instruments bores. J. Acoust. Soc. Amer., 31(2):137$146,1959$.

[16] E. Moers and J. Kergomard. On the cutoff frequency of clarinet-like instruments. geometrical versus acoustical regularity. Acta Acustica u. Acustica, 97(6):984 996, 2011.

[17] W. H. Wittrick and F. W. Williams. Natural frequencies of repetitive structures. Quart. J. Mech. and Applied Math., XXIV(3):285-308, 1971.

[18] S. Nicaise. Some results on spectral theory over networks applied to nerve impulse transmission. In C. Brezinski, editor, Lecture notes in mathematics, pages 533-541. 1985.

[19] J. Von Below. A characteristic equation associated to an eigenvalue problem on $c^{2}$ networks. Linear algebra and its applications, 71:309-325, 1985.

[20] J. L. Lagnese, G. Leugering, and E. J. P. G. Schmidt. Modelling, analysis and control of dynamic elastic multilink structures. Birkhaüser, 1994.

[21] G. Le Vey. Graph models of wind instruments : computing the natural frequencies of some elementary ducts. In International Symposium on Musical Acoustics, ISMA'2014, Le Mans, France, July 2014. 
[22] J. P. Dalmont and G. Le Vey. New lattices of sound tubes with harmonically related eigenfrequencies. In International Symposium on Musical Acoustics, ISMA’2014, Le Mans, France, 2014.

[23] G. Le Vey and J.F. Petiot. Graph-based model optimization of wind instruments : preliminary results. In International Symposium on Musical Acoustics, ISMA’2014, Le Mans, France, July 2014.

[24] A. Katchalov, Y. Kurylev, and M. Lassas. Inverse boundary spectral problems. Chapman \& Hall/CRC, 2001.

[25] J. W. Wilson. Introduction to graph theory. Academic Press, 1972.

[26] T. Hélie. Unidimensional models of acoustic propagation in axisymmetric waveguides. J. Acoust. Soc. Amer., 114(5):2633-2647, 2003.

[27] G. Szegö. Orthogonal polynomials, volume XXIII of Colloquium publications. American Mathematical Society, Providence, R.I. (1939), $4^{\text {th }}$ edition, 1975.

[28] A. Kirsch. an Introduction to the mathematical theory of inverse problems, volume 120 of Applied mathematical sciences. Springer-Verlag, 1996.

[29] G. Le Vey. Optimal control theory : a method for the design of wind instruments. Acta Acustica u. Acustica, 96:722-732, 2010.

[30] https://www.youtube.com/watch?v=8A4UmXDzDVU.

[31] J.G. Roederer. The physics and psychophysics of music, an introduction. Springer Verlag, 4th edition, 2009. 